\title{
SYNERGETIC USE OF SENTINEL-1 AND SENTINEL-2 DATA FOR EXTRACTION OF BUILT-UP AREA IN A ROCKY DESERT OASIS, EXAMPLE FOR DRAA TAFILALT, SOUTH-EAST OF MOROCCO
}

\author{
L. Eddahby ${ }^{1 *}$, A.A. Kozlova ${ }^{2}$, M.A. Popov ${ }^{2}$, N.S. Lubskiy ${ }^{2}$, D. Mezzane ${ }^{3}$, I. Lukynchuk ${ }^{4}$, A. Larabi ${ }^{1}$ and H. Ibouh ${ }^{5}$
}

${ }^{1}$ Laboratory LIMEN “Water Resources \& Information Technology”, Mohammadia School of Engineers, BO 765 Agdal, Rabat, Morocco (*DEVS/DSP in the ANDZOA, Rabat, Morocco) - eddahbylhou1@gmail.com ;

${ }^{2}$ Scientific Centre for Aerospace Research of the Earth, Kiev, Ukraine - casre@casre.kiev.ua ;

${ }^{3}$ LMCN, Faculty of Sciences and Techniques, Cadi Ayyad University, Marrakesh, Morocco;

${ }^{4}$ LPMC, Picardie Jules Verne University, Amiens, France ;

${ }^{5}$ LGSE, Faculty of Sciences and Techniques, Cadi Ayyad University, Marrakesh, Morocco - h.ibouh@uca.ma;

\section{Commission VI, WG VI/4}

KEY WORDS: Build-up area, Sentinel-1, Sentinel-2, Neural Net Classification, Remote sensing, Tafilalet, Morocco.

\begin{abstract}
:
Synthetic Aperture Radar (SAR) is an active remote sensing technique capable of providing high-resolution imagery independent from daytime and to great extent unimpaired by weather conditions. Unlike the passive remote sensing active radars receive its' own reflected signal. Features of received signal make able to obtain additional information about surface objects and covers. Because of signal, double reflecting upon vertical surfaces like walls, it become common to study urbanized areas using SAR data. Because of mostly similar spectral characteristic of the typical buildings' roofs and sandy soils, that are distinguishing for Morocco, classification using visible and NIR multispectral remote sensing data is complicated. Thus, SAR data processing technique is rather useful while application to deserted area studying and urbanized areas identification.
\end{abstract}

\section{INTRODUCTION}

Novel Sentinel satellites of the Earth observation missions provided by European Space Agency raised a new wave of discussions on synergism of multiple sensor data application for urban extraction in general and radar and optical sensors in particular (Kozlova, 2018; Strozzi et al., 2018 and Van et al., 2018). Although challenged by spatial and spectral heterogeneity of urban environments, optical and synthetic aperture radar (SAR) imagery seem to be suitable sources of reliable information about the multiple facets of urban environments (Corbane, 2008). However, demonstrating excellent results in urban extraction over large cities and agglomerations (Ban, 2017), insufficient attention was paid to the recognition of towns and settlements in complex landscapes (Khadim et al., 2016).

The purpose of the presented study is to derive a methodological framework to integrate Sentinel-1 and Sentinel2 imagery for accurate mapping of built-up areas in an oasis of rocky desert by integrating the advantages of radar and optical imagery.

\section{STUDY AREA AND METHODS}

\subsection{Study area}

Tafilalt oasis was selected as a study area. It is the largest Saharan ones in South-East of Morocco. Tafilat is a geographic region in the western part of Moroccan Kingdom which includes several oases. The region comprises the fortified villages of Erfoud, Arab Sebbah du Ziz, Rissani, Seffalat, Aoufous, and Jorf, together with palm groves stretching $50 \mathrm{~km}$ along the Wadi Ziz river. Relates to the province of Errachidia, the center is located in the Erfoud town. The plain with hills 500-1000 $\mathrm{m}$ high is located in the basin of the Geris and Wadi Ziz rivers flowing from the High Atlas Moutain.

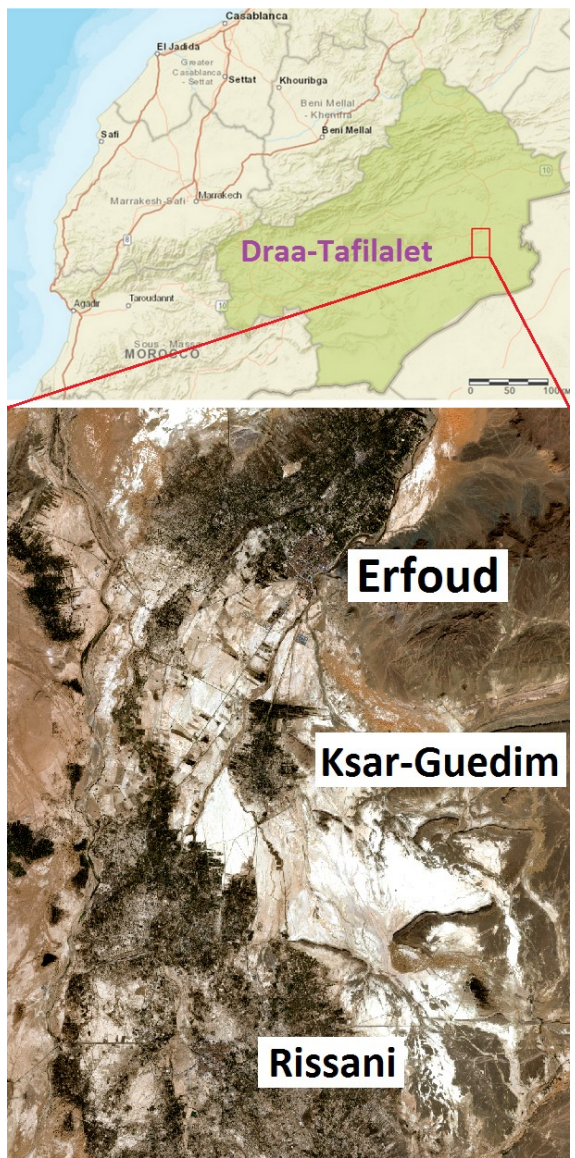

Figure 1. Study area 


\subsection{Material and Methods}

Sentinel-2A level 2 multispectral image over the study area as well as two Sentinel 1A images (Level-1 SLC) forming an interferometric pair were downloaded from the ESA's Sentinel Scientific Hub (https://scihub.copernicus.eu ). SAR images were acquired on November 6th and 18th 2017, whereas multispectral image was available only for November 6th, 2017.

Data pre-processing of the Sentintinel-1 and Sentinel-2A images was performed using the SNAP Sentinel Application Platform toolbox. Standard processing methods were used to calibrate both Sentinel-1 SLC images to, simultaneously, interferometric coherence was also calculated. Synthetic Aperture Radar (SAR) is a coherent active microwave imaging method in remote sensing used for mapping the scattering properties of the Earth's surface.

Coherence, as a measurement of stability of interferograms of SAR interferometry (InSAR), contains useful information on changes of geophysical phenomena. (Zebker, 1992). Coherence $(\gamma)$ is a measurement of the stability of the interferogram that is used to measure displacement or derive a digital elevation model (DEM) of the surface using the InSAR technique. Calculation of the coherence is based on equation (1) (Lee, 2003):

$$
\gamma=\frac{\left\langle I_{1} I_{2}^{*}\right\rangle}{\sqrt{\left\langle\left|I_{1}\right|^{2}\right\rangle\left\langle\left|I_{2}\right|^{2}\right\rangle}}
$$

Where $\gamma$ is the estimated coherence with a value between 0 and 1. $I_{1}$ and $I_{2}$ are the complex matrices of backscattering coefficient of complex images 1 and 2 , respectively. ${ }^{*}$ denotes complex conjugate matrix. \langle\rangle indicates ensemble average.

In order to produce homogenous, filtered from speckle noise and terrain-rectified images, SNAP standard methods of TOPSAR deburst, multilook parametrization for both VV and VH polarization images and Range Doppler orthorectification were also applied. Pre-processed Sentinel-2A (Figure 2) and Sentinel-1a co-registered into 20-band image is shown in Figure 3.

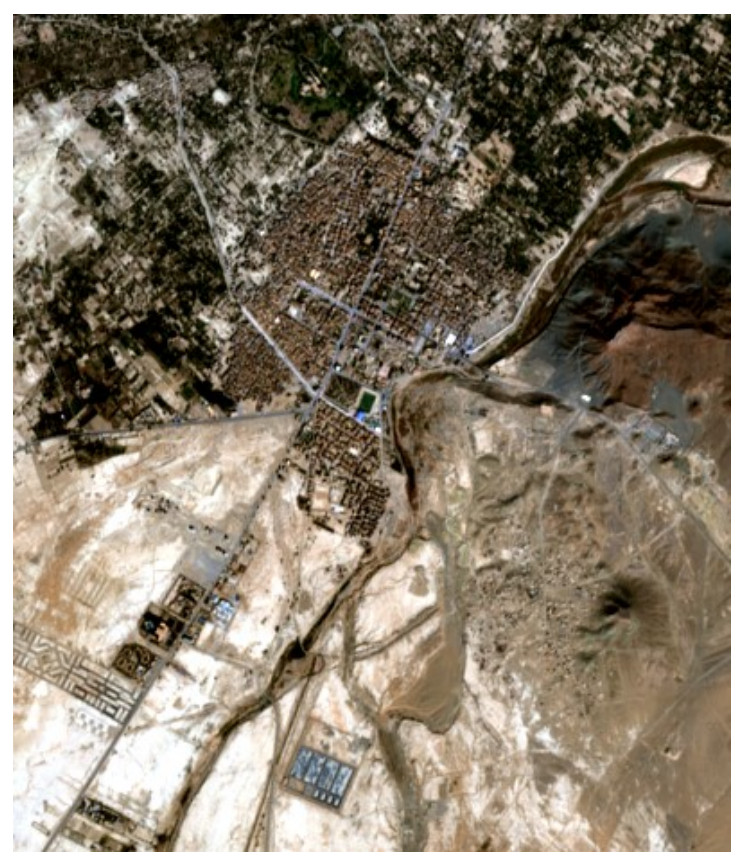

Figure 2. Erfoud town satellite image: Sentinel-2 visible data

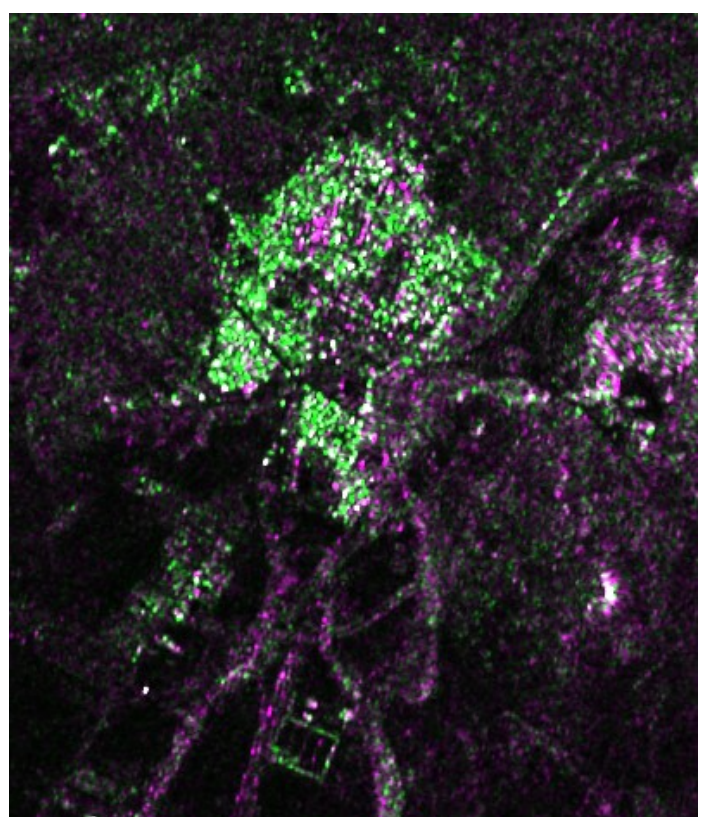

Figure 3. Erfoud town satellite image Sentinel-1 SAR data $\mathrm{VH}+\mathrm{VV}+\mathrm{VH} \sigma 0$ values RGB colour composite

For removing high noise rate of the SAR signal, which comes from rocky area and ridges digital elevation model (DEM) SRTM also was included to data stack (Figure 4). In addition, DEM is used for excluding elevation differences between urbanized areas and others, which occurs due to buildings' height.

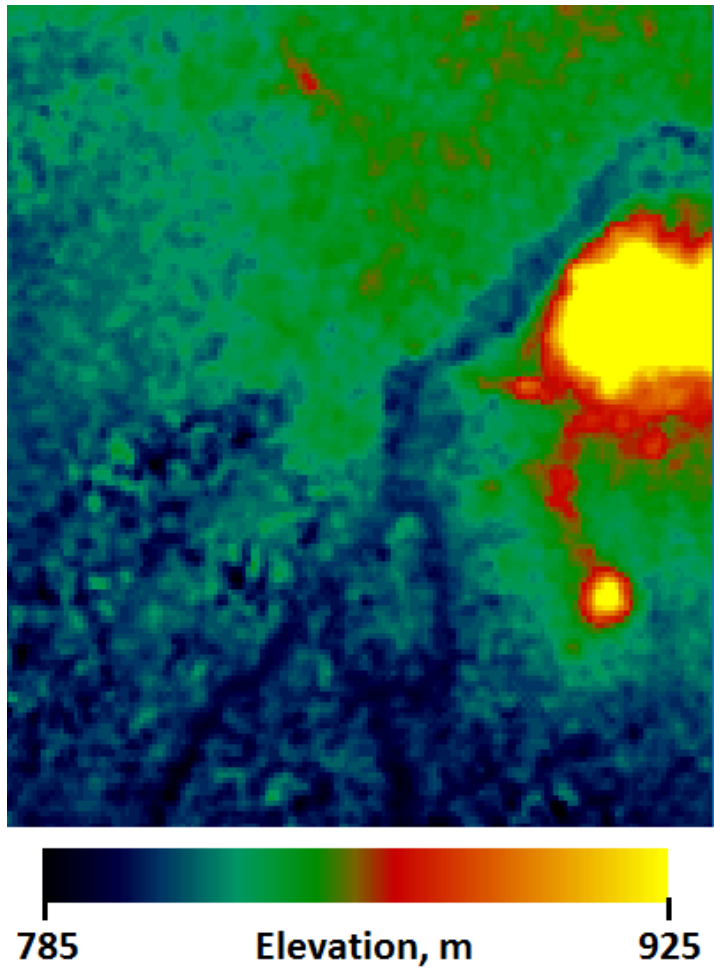

Figure 4. SRTM digital elevation model of the Erfoud town area

Coherence data demonstrated high values $(0,7-0,9)$ for all sandfree areas. Due to dynamic elevation changes on sandy areas, 
low coherence is normal for it. Coherence image for Erfoud town area demonstrated on Figure 5.
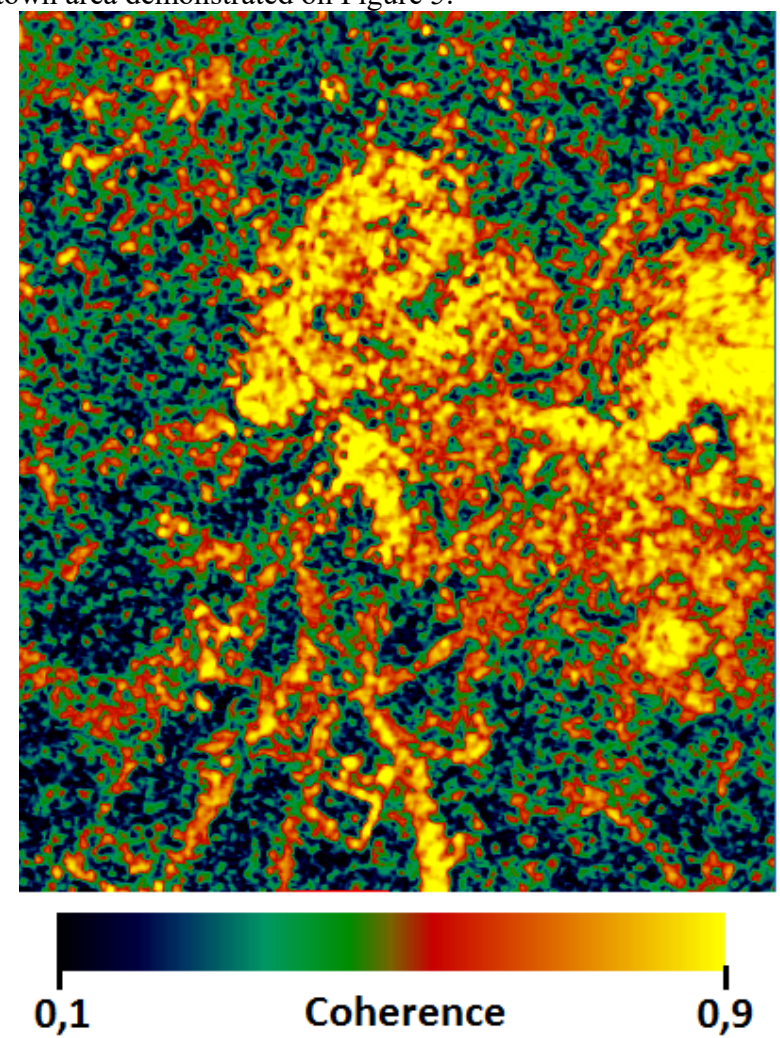

Figure 5. Image showing the Erfoud town SAR coherence data.

The image of the study area was classified into five classes: artificial surfaces, such as roofs, three types of sands and soils, depending on the spectral brightness and rocky ridges. Supervised classification by Neural Net has been performed.

The Neural Net technique uses standard backpropagation for supervised learning. Learning occurs by adjusting the weights in the node to minimize the difference between the output node activation and the output. The error is backpropagated through the network and weight adjustment is made using a recursive method (Richards, 1999).

Initially, it is necessary to create a training sample, which consists of small polygons and points, so-called regions of interest (ROI), which within its' boundaries include only pixels of the certain class. The purity of the sample is the determining factor for the classification accuracy. All available remote sensing data is used for the study area classification: multispectral Sentinel-2 imagery in the spectral range of 0.45 $2.3 \mu \mathrm{m}$, Sentinel-1 radar interferometry data (Haas, 2017), and the SRTM digital elevation model.

\subsection{Classification efficiency estimation}

The result of classification is a spatial distribution of territories that correspond to its class from the training sample. Representative fragment of the obtained classification is shown in Figure 6.

Classification accuracy verification is carried out by forming new regions of interest that are consist of a single point samples randomly distributed with a further visual correspondence examination of each sample to the submitted class on the original multispectral image. The number of verification points for each class is chosen proportionally to its square in the classification results. Out of 170 verification points 140 are corresponded to its class, which signify $82.4 \%$ classification accuracy. The result of urban extraction precision estimation, based on random sample method, is $77,5 \%$ : from 200 random urban class samples 155 occupied urbanized area.

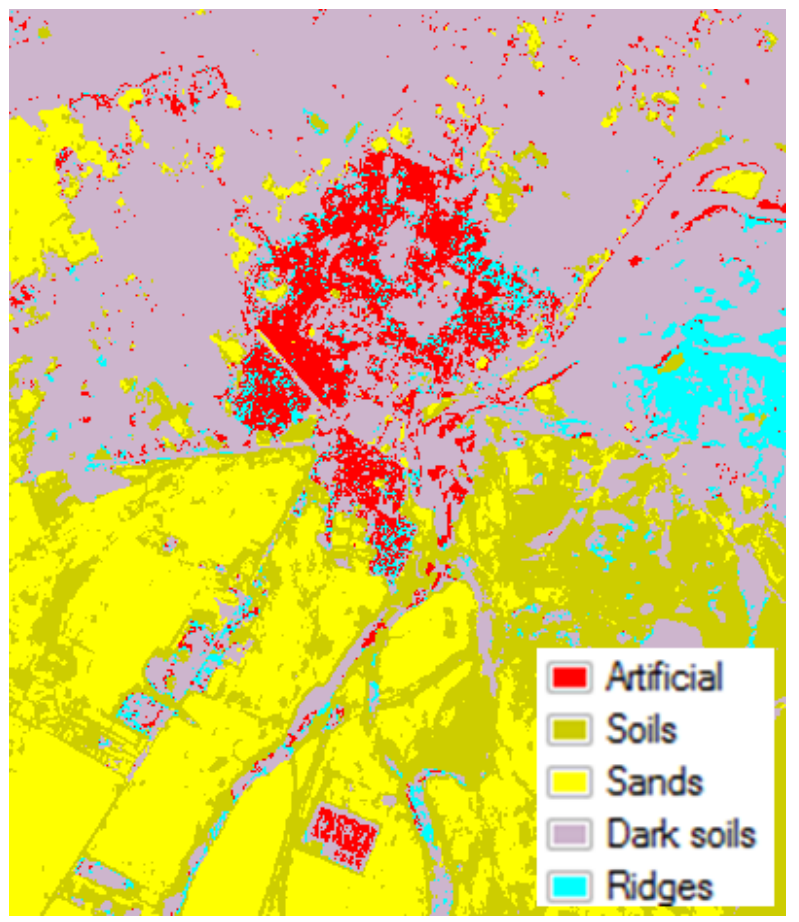

Figure 6. Erfoud town satellite imagery classification results

\section{CONCLUSIONS}

This research demonstrates that synergetic use of Sentinel-1 SAR and Sentinel-2 MSI data for build-up areas extraction is very promising for analysis of urban development within morphologically complex landscape of a rocky desert oasis.

\section{REFERENCES}

Ban, Y., Webber, L., Gamba, P., Paganini, M., 2017. EO4Urban: Sentinel-1A SAR and Sentinel-2A MSI data for global urban services. In: Proceedings of the 2017 Joint Urban Remote Sensing Event (JURSE) doi:10.1109/jurse.2017.7924550.

Corbane, C., Faure, J.F., Baghdadi, N., Villeneuve, N., Petit, M., 2008. Rapid Urban Mapping Using SAR/Optical Imagery Synergy. Sensors, 8, p. 7125-7143.

Haas, J., Banб Y., 2017. Sentinel-1A SAR and sentinel-2A MSI data fusion for urban ecosystem service mapping. Remote Sensing Applications: 8, p. 41-53.

Kadhim N., Mourshed M. and Bray M. 2016 Advances in remote sensing applications for urban sustainability. EuroMediterranean Journal for Environmental Integration, December 2016, 1:7

Kozlova, A.A., Khyzhniak, A.V., Piestova, I.A., Andreiev, A.A., 2018. Synergetic use of Sentinel-1 and Sentinel-2 data for analysis of urban development and green spaces. Abstracts of the XVIIth International Conference "Geoinformatics: 
Theoretical and Applied Aspects", 14 - 17 May 2018, Kiev, Ukraine.DOI: 10.3997/2214-4609.201801846

Lee, J.S., Cloude, S.R., Papathanassiou, K.P., Grunes, M.R., and Woodhouse, I.H., 2003. Speckle filtering and coher-ence estimation of polarimetric SAR interferometry data for forest applications. IEEE Transactions on Geoscience and Remote Sensing, 41(10), p. 2254-2263

Richards, J.A., 1999. Remote Sensing Digital Image Analysis. Berlin: Springer-Verlag, 240 pp.

Strozzi T., Antonova S., Günther F., Mätzler E., Gonçalo VieiraG., Wegmüller U., Westermann S. and Bartsch A. 2018. Sentinel-1 SAR Interferometry for Surface Deformation Monitoring in Low-Land Permafrost Areas Remote Sens. 2018, 10(9), 1360; https://doi.org/10.3390/rs10091360

Van, T.T.; Tran, N.D.H.; Bao, H.D.X.; Phuong, D.T.T.; Hoa, P.K.; Han, T.T.N. 2018. Optical Remote Sensing Method for Detecting Urban Green Space as Indicator Serving City Sustainable Development. Proceedings 2018, 2(3), 140; https://doi.org/10.3390/ecsa-4-04932

Zebker, H.A., Villasenor, J., 1992. Decorrelation in interferometric radar echoes. IEEE Transactions on Geoscience and Remote Sensing, 30(): p. 950-959. 